\title{
High-quality bilayer graphene grown on softened copper foils by atmospheric pressure chemical vapor deposition
}

\author{
Qiao Chen ${ }^{1,2}$, Qiyang Song ${ }^{1}$, Xin $\mathrm{Yi}^{1}$, Qiao Chen ${ }^{1}$, Wenjia Wu ${ }^{1}$, Meirong Huang ${ }^{2}$, Chuanwen Zhao ${ }^{1}$, \\ Shun Wang ${ }^{1,3^{*}}$ and Hongwei Zhu ${ }^{2^{*}}$
}

\begin{abstract}
Bilayer graphene (BLG) shows great application prospect and potential in next-generation electronics because of its unique electrical and mechanical properties. However, the scalable synthesis of large-area high-quality BLG films is still a great challenge, despite the maturity of chemical vapor deposition (CVD) technique. In this study, we report a robust method to grow BLGs on flat, softened $\mathrm{Cu}$ foils by atmospheric pressure CVD. A moderate amount of residual oxygen accelerates the growth of BLG domains while suppressing the formation of multilayers. Raising the nucleation density at low hydrogen pressure efficiently increases the film continuity. Based on the optimized CVD process, the growth of graphene films on $4 \times 4 \mathrm{~cm}^{2} \mathrm{Cu}$ foils with an average BLG coverage of $76 \%$ is achieved. The morphology and structure characterizations demonstrate a high quality of the BLG. Dual gate field-effect transistors are investigated based on AB-stacked BLG, with a tunable bandgap and high carrier mobility of up to $6790 \mathrm{~cm}^{2} \mathrm{~V}^{-1} \mathrm{~s}^{-1}$ at room temperature.
\end{abstract}

Keywords: graphene, bilayer, chemical vapor deposition, $\mathrm{Cu}$ foil

\section{INTRODUCTION}

The realizations of tunable bandgap, unconventional superconductivity and ultrahigh hardness in bilayer graphene (BLG) make it fascinating for fundamental research as well as emerging electronic applications [1-3]. A scalable approach to synthesizing high-quality BLG is of great importance. Large-area single-layer graphene (SLG) can be achieved by chemical vapor deposition (CVD) on copper [4-6], thanks to the negligible solubility of carbon atoms in $\mathrm{Cu}$ and the self-limited growth me- chanism [6,7]. However, it becomes a great challenge for BLG, owing to the complex manufacturing processes brought by the additional layers of graphene. Strategies such as using hazard gas (e.g., $\mathrm{H}_{2}$ ) with high partial pressure [8-10], edge etching of the top graphene layer [11], and using alloy or multicomponent catalyst substrates [12-15] have been explored to synthesize BLG. Nevertheless, it is still imperative to develop a practical and industrially viable method for BLG preparation.

BLG can be prepared based on small ad-layered graphene (ALG), which is often formed beneath SLG synthesized on $\mathrm{Cu}$ [8-11,15-17]. Efforts have been made to investigate the nucleation and growth kinetics of ALG [18-21]. The understanding of the growth mechanism of ALG facilitates the layer uniformity of desired large-area products. For example, continuous SLG films have been achieved by simultaneous growth of SLG on both sides of the suspended $\mathrm{Cu}$ foil, which suppresses the ALG growth in a normal CVD process [22]. Different from SLG growth, BLG growth requires two unequal sides of $\mathrm{Cu}$. It is essential to ensure the bulk diffusion of carbon atoms to the BLG growth side of the $\mathrm{Cu}$ foil, meanwhile to suppress graphene growth on the other side. Strategies such as the adoption of a $\mathrm{Cu}$ pocket to limit the carbon concentration inside the pocket have been successfully employed to grow BLG domains on the exterior surface $[16,19]$. However, drawbacks like low carbon diffusivity of the inside atmosphere and the changeable gap size of crimped edges undoubtedly limit the scalable use of $\mathrm{Cu}$ pockets. Furthermore, when both sides of the $\mathrm{Cu}$ foil are completely covered by graphene, forming a barrier for

\footnotetext{
${ }^{1}$ MOE Key Lab of Fundamental Physical Quantities Measurement \& Hubei Key Lab of Gravitation and Quantum Physics, School of Physics, Huazhong University of Science and Technology, Wuhan 430074, China

${ }^{2}$ State Key Lab of New Ceramics and Fine Processing, School of Materials Science and Engineering, Tsinghua University, Beijing 100084, China

${ }^{3}$ Shenzhen Huazhong University of Science and Technology Research Institute, Shenzhen 518057, China

* Corresponding authors (emails: hongweizhu@tsinghua.edu.cn (Zhu H); shun@hust.edu.cn (Wang S))
} 
carbon diffusion, BLG stops to grow [19,23]. Therefore, the growth rate should be fast enough to prepare large BLG. BLG domains with size of hundreds of microns have been achieved after $6 \mathrm{~h}$ of growth with the assistance of oxygen to accelerate the carbon bulk diffusion process [16]. Another key factor for the preparation of uniform BLG is to suppress the growth of tri-layer graphene (TLG) and thicker multilayer graphene (MLG) $[21,24,25]$. As ALG often forms at the same initial nucleation centers and grows simultaneously with the first graphene layer [20], it is quite difficult to completely avoid undesired TLG or MLG.

In this work, we developed a robust and efficient method for atmospheric pressure CVD (AP-CVD) of high-quality BLG on $\mathrm{Cu}$ foils. The flat $\mathrm{Cu}$ foil was gradually softened and stacked onto the quartz tube/carrier surface during a designed heating process. The quartz significantly contributed to the formation of large BLG domains on the top side of $\mathrm{Cu}$ foil by ensuring the difference on two sides of $\mathrm{Cu}$ foil, suppressing the backside graphene nucleation density, and providing a continuous supply of oxygen during the CVD process. Ar flow was adopted to exhaust residual oxidative species in the APCVD chamber. Excessive time of Ar flow suppressed the growth of BLG, while shortening the Ar flow time greatly promoted the growth of excess TLG and MLG. The results were associated with the increased oxidation of $\mathrm{Cu}$ foil by system residual oxygen. Low hydrogen flow rate was efficient to promote the nucleation of BLG. With optimized parameters, continuous graphene films with average BLG covering of $76 \%$ were achieved. Detailed morphology and structure characterizations confirmed that the obtained BLG was of high quality. The ABstacked BLGs exhibited tunable bandgaps with carrier mobility up to $6790 \mathrm{~cm}^{2} \mathrm{~V}^{-1} \mathrm{~s}^{-1}$ at room temperature observed in the dual-gate field effect transistors (FETs).

\section{EXPERIMENTAL SECTION}

\section{Growth of BLG}

The synthesis process was carried out in a thermal CVD furnace with a quartz tube $(170 \mathrm{~cm}$ in length, $5 \mathrm{~cm}$ in diameter) at atmospheric pressure [26,27]. The quartz tube was sealed by two flanges to maintain the reactive atmosphere and avoid contamination. $\mathrm{Cu}$ foils $(25 \mu \mathrm{m}$, 99.8\%, Alfa Aesar) were used as the catalytic substrates. Acetone (99.5\%, Peking Reagent) was used as the carbon source. The acetone was accurately fed to the CVD system with a sustained and controlled release approach, as described in our previous study [27]. A $4 \times 4 \mathrm{~cm}^{2} \mathrm{Cu}$ foil was placed at the center reaction zone on a semicircular quartz carrier (see Fig. S1a). Ar $\left(300 \mathrm{~mL} \mathrm{~min}^{-1}\right)$ was flowing for 40-300 min to exhaust the residual oxygen in the AP-CVD system. The furnace was heated to the reaction temperature $\left(1040^{\circ} \mathrm{C}\right)$ at a speed of $10^{\circ} \mathrm{C} \mathrm{min}^{-1}$. It took $100 \mathrm{~min}$ to heat up the $\mathrm{Cu}$ foil, as shown in the schematic diagram (Fig. 1a). The growth time was $60 \mathrm{~min}$ with $\mathrm{H}_{2}\left(10-20 \mathrm{~mL} \mathrm{~min}^{-1}\right)$ and $\mathrm{Ar}\left(300 \mathrm{~mL} \mathrm{~min}^{-1}\right)$ flows. The $\mathrm{Cu}$ foil was rapidly cooled down after graphene growth.

\section{Growth of SLG}

As shown in Fig. S2, the $\mathrm{Cu}$ foil was rapidly pushed into the reaction zone when the furnace reached $1040^{\circ} \mathrm{C}$. Besides the fast heating up process of the $\mathrm{Cu}$ foil (about $1 \mathrm{~min}$ ), other growth parameters were the same as the preparation of BLG.

\section{Growth of MLG}

The quartz carrier was replaced by a quartz shelf support (Fig. S3) to maintain equal carbon precursor concentration on two sides of the $\mathrm{Cu}$ foil. Other growth parameters remained the same as the growth of BLG.

\section{Transfer of graphene}

Graphene films grown on $\mathrm{Cu}$ foils were wet-transferred onto $\mathrm{SiO}_{2} / \mathrm{Si}\left(300 \mathrm{~nm}\right.$ thick $\left.\mathrm{SiO}_{2}\right)$ substrates by etching $\mathrm{Cu}$ with polymethylmethacrylate (PMMA) protection for subsequent characterizations or applications. The PMMA layer was removed by immersing in acetone and isopropanol.

\section{Structural characterizations}

The as-prepared graphene samples were characterized by scanning electron microscopy (SEM, MERLIN VP Compact, Carl Zeiss), transmission electron microscopy (TEM, JEM-2100F, JEOL), Raman spectroscopy (Horiba Evolution, $532 \mathrm{~nm}$ laser excitation), and atomic force microscopy (AFM, Dimension Edge, BRUKER, Tapping mode). Optical images were taken by an Axio scope A1 optical microscope and a Nikon Eclipse Ni-U optical microscope.

\section{Fabrication and measurements of BLG-based FETs}

BLG-based FETs were fabricated on $300 \mathrm{~nm} \mathrm{SiO}_{2} / \mathrm{Si}$ substrates using photolithography (ATD 1000). $\mathrm{Cr}(5 \mathrm{~nm}) / \mathrm{Au}(50 \mathrm{~nm})$ were deposited as contact electrodes using electron-beam evaporation (FU-121PEB). The channel length and width were set as 5 and $10 \mu \mathrm{m}$, respectively. The devices were thermally annealed in va- 

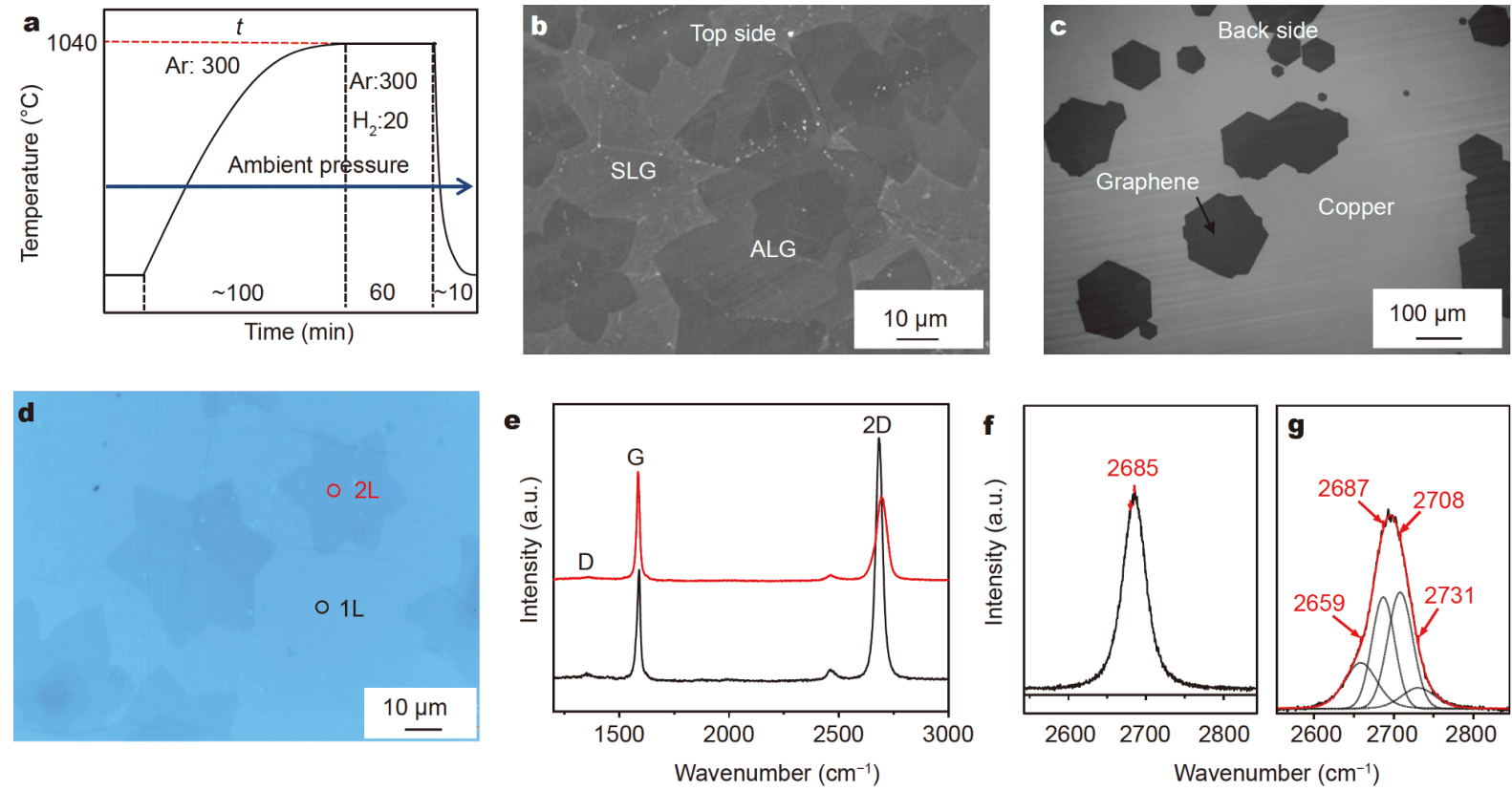

Figure 1 (a) Schematic diagram of the time-dependent AP-CVD parameters. SEM images of graphene grown on (b) top side and (c) back side of the $\mathrm{Cu}$ foil. (d) Optical image of the top side graphene transferred on $\mathrm{SiO}_{2} / \mathrm{Si}$. (e) Corresponding Raman spectra of SLG (black) and BLG (red) recorded at the spots marked in (d). Raman 2D bands of (f) SLG and (g) BLG. The peak of BLG is well fitted with four Lorentzian functions (black dash lines).

cuum at $150^{\circ} \mathrm{C}$ for $40 \mathrm{~min}$ to improve the contact between BLG and the electrodes. Ionic liquid, 1-ethyl-3methylimidazolium bis(trifluoromethanesulfonyl)imide (99.9\%, Solvionic) was used as the top gate dielectric material. A drop of ionic liquid was cast onto the substrate with graphene and the coplanar electrode to form the top gate. Electrical characterizations were performed with a Keysight B1500A semiconductor analyzer at room temperature. The effect of the dual-gate was investigated by measuring the current in vacuum $\left(3 \times 10^{-3}\right.$ Torr $)$ under a fixed drain bias $(10 \mathrm{mV})$. The mobility of charge carriers was extracted with the equation:

$\mu=\frac{\mathrm{d} I_{\mathrm{DS}}}{\mathrm{d} V_{\mathrm{BG}}} \cdot \frac{L}{W} \cdot \frac{1}{C_{\mathrm{G}} V_{\mathrm{DS}}}$,

where $I_{\mathrm{DS}}$ is the source-drain current, $V_{\mathrm{BG}}$ is the back-gate voltage, $L$ and $W$ are the device channel length and width, $C_{\mathrm{G}}$ is the gate capacitance per unit area $\left(1.15 \times 10^{-8} \mathrm{~F} \mathrm{~cm}^{-2}\right)$, and $V_{\mathrm{DS}}$ is the voltage between source and drain electrodes.

\section{RESULTS AND DISCUSSION}

Carrier gas Ar was commonly used to exhaust air in the AP-CVD system. In previous studies, oxygen had been intentionally introduced and showed complicated effects on graphene growth [16,28-32], such as the control of nucleation density, acceleration of growth rate, alteration of domain shape and modulation of number of layers. However, the amount of residual oxidative species in the CVD chamber and its effects on graphene growth are not

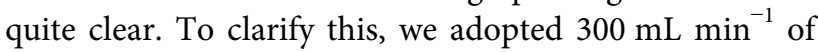
Ar to degas the AP-CVD system for different times $(t)$ before the growth of graphene. Generally, Ar flow started simultaneously with the furnace heating (for example, $t=$ $100 \mathrm{~min}$, denoted as Ar-100 min).

Continuous graphene films were obtained on the top side of the $\mathrm{Cu}$ foil following the growth procedure illustrated in Fig. 1a. However, the back side of $\mathrm{Cu}$ foil was only covered with partially grown graphene (Fig. S1b, c). As observed by SEM, SLG film accompanying ALG sheets were formed on the top side of the Cu foil (Fig. 1b), while discrete domains were found on the back side of $\mathrm{Cu}$ foil (Fig. 1c). Optical image of the top side graphene film transferred on $\mathrm{SiO}_{2} / \mathrm{Si}$ substrate shows large BLG domains of about $50 \mu \mathrm{m}$ (Fig. 1d). Raman spectrum of the as-prepared graphene film was recorded. As shown in Fig. 1e, the disorder-activated D peak $\left(\sim 1350 \mathrm{~cm}^{-1}\right)$ is almost negligible compared with the sharp G $\left(\sim 1583 \mathrm{~cm}^{-1}\right)$ and 2D $\left(\sim 2690 \mathrm{~cm}^{-1}\right)$ peaks, which demonstrates high crystallinity with minimal defects $[33,34]$. The single Lorentzian profile of $2 \mathrm{D}$ band with a full width at half-maximum (FWHM) of $35 \mathrm{~cm}^{-1}$ (Fig. 1f) and high-intensity ratio of $2 \mathrm{D} / \mathrm{G}\left(I_{2 \mathrm{D}} / I_{\mathrm{G}}>2\right)$ indicates the typical monolayer structure for the black spot marked in 
Fig. 1d. Raman spectrum of the red spot marked in Fig. 1d shows the typical feature of AB-stacked BLG. The FWHM of the 2D peak of the red spot marked in Fig. 1d is about $50 \mathrm{~cm}^{-1}$ and the $2 \mathrm{D}$ band intensity is significantly reduced. Moreover, the $2 \mathrm{D}$ band is well fitted with four Lorentzian peaks centered at 2695, 2687, 2708 and $2731 \mathrm{~cm}^{-1}$, which shows a typical AB-stacked BLG feature [33].

To understand the growth mechanism of BLG, a control experiment was performed by setting a fast heating process of $\mathrm{Cu}$ foil without any changes of other growth parameters, as shown in Fig. S2a. Interestingly, it turns out that uniform SLG film without obvious ALG forms on the top side of $\mathrm{Cu}$ foil (Fig. S2b). In addition, the back side of $\mathrm{Cu}$ foil is also nearly completely covered by SLG, as shown in Fig. S2c. The results demonstrate that the heating process of $\mathrm{Cu}$ foil has significant effects on the morphology of the as-prepared graphene. As discussed above, the key point of uniform SLG synthesis is the simultaneous growth of graphene on both sides of $\mathrm{Cu}$ foil [22]. Here, the fast heating process caused a rapidly starting of graphene growth once the $\mathrm{Cu}$ foil reached the reaction zone, when the conditions on both sides of the $\mathrm{Cu}$ foil were almost identical (Fig. S2d). For the slowly heating process, the $\mathrm{Cu}$ foil was gradually softened onto the quartz tube/carrier, as shown in Fig. 2a. The back side of $\mathrm{Cu}$ foil was consequently stacked on the quartz surface when it reached the growth temperature before the growth begins. This generated two unequal sides of the $\mathrm{Cu}$ foil, which is the key to prepare high-quality BLG. As further confirmed in Fig. S3, when a quartz shelf support was used to suspend $\mathrm{Cu}$ foil forming equal atmosphere on two sides with other growth parameters remaining un- changed, only inhomogeneous graphene film with thick MLG formed on both sides of the $\mathrm{Cu}$ foil (Fig. S4).

Furthermore, the backside quartz tube/carrier is similar to a deliberately placed oxide support according to previous studies $[23,31,35]$. The backside quartz is determined to suppress the backside nucleation density of graphene, although the mechanism is debatable on the forming channel structure or oxygen release from the quartz $[31,35]$. This is why only discrete graphene domains are on the back side of $\mathrm{Cu}$ foil heated slowly with furnace. There are mainly two types of growth mechanisms of BLG: (i) a small fraction of carbon on the surface of $\mathrm{Cu}$ directly diffuses beneath the top graphene layer to nucleate BLG; (ii) carbon on the slower graphene growth side diffuses through the $\mathrm{Cu}$ to form BLG on the other side. Because of the barrier of top graphene layer, the growth rate of BLG based on surface diffusion is much lower $[9,20]$. Bulk diffusion hence dominates the growth of BLG. According to previous results, oxygen plays a key role for promoting BLG synthesis by accelerating the bulk diffusion process [16]. The backside quartz is found providing a continuous supply of oxygen during the CVD growth [31], which further contributes to the growth of the BLG domains.

Generally, the quartz tube/carrier as a backside oxide support suppresses the nucleation of the back-side graphene, and promotes carbon diffusing to the top side, significantly inducing large BLG growth on the top side of the flat $\mathrm{Cu}$ foil. In addition, due to the oxidation of $\mathrm{Cu}$ foil during the heating by residual oxidative species in AP-CVD system [36-38], the oxygen released from copper oxides also participates in the synthetic processes and generates huge impacts on the graphene synthesis.
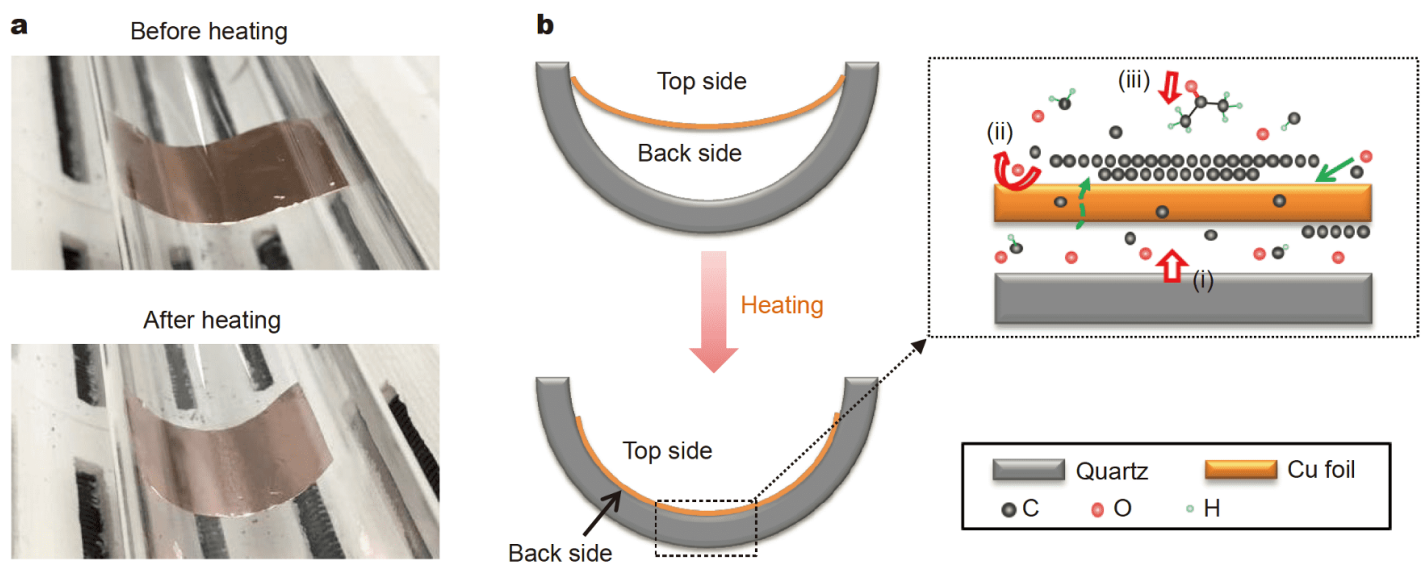

Figure 2 (a) Photographs of a $4 \times 4 \mathrm{~cm}^{2} \mathrm{Cu}$ foil before and after heating. (b) Schematic illustrations of the growth mechanism for the BLG with the assistance of the back-side quartz tube/carrier. 
Besides, the oxygen atoms in carbon source (acetone) are non-negligible, which might facilitate the crystallization of BLG [26,27]. Schematic illustration of the proposed mechanism of BLG growth is displayed in Fig. $2 \mathrm{~b}$ : the solid green line with arrow represents the surface diffusion and the dashed green line with arrow represents the bulk diffusion of carbon atoms, while the red arrows indicate (i) the oxygen supplied by quartz, (ii) the surface copper oxide decomposition and (iii) the oxygenated functional groups in carbon source, respectively. Oxygen released from copper oxides helps graphene growth by promoting hydrocarbon dissociation $[9,16,30]$. Cu foils are oxidized at different levels with different times of Ar flow to exhaust the system residual oxygen, as illustrated in Fig. S5. The color changed gradually from yellow to purple, indicating increased oxidation of $\mathrm{Cu}$ with shortened time of Ar flow. X-ray photoelectron spectroscopy (XPS) characterizations (Fig. S6) indicate that the copper oxides on the surface of $\mathrm{Cu}$ foil are mainly $\mathrm{CuO}$. When the Ar flow time decreased from 300 to $40 \mathrm{~min}$, the corresponding oxidation ratio of $\mathrm{Cu}$ increased from $2 \%$ to $46 \%$ (Table S1). Especially, when the Ar flow time was less than $100 \mathrm{~min}$, the $\mathrm{Cu}$ oxidation ratio increased rapidly.

The increased amount of copper oxides was expected to further promote the growth of BLG. As shown in Fig. 3a, shortening the Ar flow time increases the amount of ALG on the top side of $\mathrm{Cu}$ foil. More carbon accumulations (strong contrast in optical images) deposited on the graphene films prepared with less Ar flow. A hexagonal
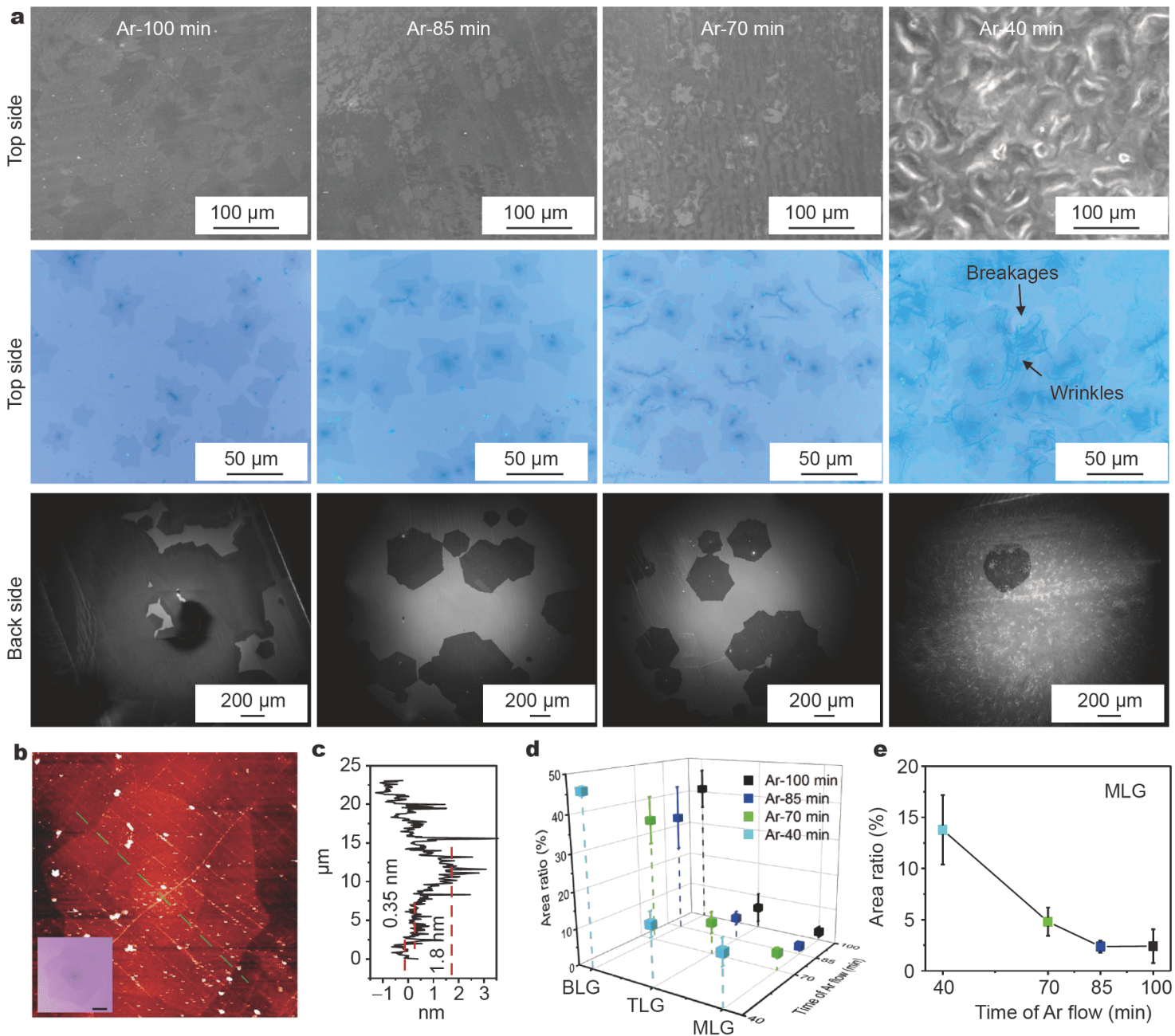

Figure 3 (a) Morphology characterizations of graphene grown with 100, 85, 70, and 40 min of Ar flow. From up to bottom, the SEM images of graphene on the top side of $\mathrm{Cu}$ foil, optical images of the top side graphene transferred on $\mathrm{SiO}_{2} / \mathrm{Si}$, and $\mathrm{SEM}$ images of graphene on the back side of $\mathrm{Cu}$ foil. (b) AFM image of a BLG domain. Inset shows the optical image (scale bar: $5 \mu \mathrm{m}$ ). (c) Corresponding height profile measured along the green dash line in (b). (d) The area percentage of BLG, TLG and MLG. (e) Correlation between the coverage of MLG and the Ar flow time. 
BLG domain of about $20 \mu \mathrm{m}$ was observed in the AFM image (Fig. 3b). The height of carbon accumulations in the center is about $2 \mathrm{~nm}$ higher than the BLG domain (Fig. 3c), which could be regarded as the undesired MLG. Moreover, with the decrease of the Ar flow time, the $\mathrm{Cu}$ foil surface becomes rougher, as shown in the SEM images. In particular, for the Ar-40 min sample the uneven morphology causes more wrinkles and breakages in the transferred graphene. Additionally, few back-side graphene domains were found for the Ar-40 min sample. Detailed statistics of the proportions of BLG, TLG and MLG are shown in Fig. 3d. The coverage of BLG are all around $45 \%$ with small variations, while the area percentage of the undesired TLG and MLG shows a clear increase with the decrease of Ar flow. As shown in Fig. 3e, the proportion of MLG is about $2 \%$ for the Ar- 100 min samples, while it approaches $15 \%$ for the Ar- $40 \mathrm{~min}$ samples. These results indicate that excessive copper oxides have little benefit for the growth of BLG, and even promote the growth of excess TLG and MLG, which sacrifices the morphology uniformity. Too much oxygen released from copper oxide promotes the pyrolysis of carbon precursors and accelerates the bulk diffusion of carbon [16]. This might cause a large amount of carbon atoms accumulating to TLG or MLG before reaching the BLG edges to form BLG.

Obviously, the optimization of the oxygen content alone has reached its limit in the growth of large-area continuous BLG. Besides accelerating the growth rate to increase the size of BLG domains, high nucleation density also plays an important role for preparing large-area BLG films. Hydrogen is commonly introduced to maintain the catalytic activity of $\mathrm{Cu}$ and meanwhile to etch disorder carbon structure on the $\mathrm{Cu}$ surface, ensuring the growth of graphene. Because of the etching effect of hydrogen on graphene at high temperature, the nucleation of graphene was also suppressed [39]. The nucleation density of graphene was low at hydrogen-rich atmosphere during the CVD process $[40,41]$. ALG was found sharing the same nucleation center with the top SLG on $\mathrm{Cu}$ [20]. To synthesize large BLG films, the hydrogen flow rate was reduced to $10 \mathrm{~mL} \mathrm{m^{-1 }}$. As shown in Fig. 4a, when the Ar flow is $100 \mathrm{~min}$, reducing the hydrogen flow rate significantly increases the nucleation of ALG. The productivity comparison of different layers of graphene grown under 20 and $10 \mathrm{~mL} \mathrm{~min}^{-1}$ of hydrogen is shown in Fig. $4 \mathrm{~b}$. The productivities of BLG, TLG and MLG were all improved at low hydrogen flow rate, and the corresponding proportion of SLG decreased by about $30 \%$. The total coverage of BLG and ALG reached over $85 \%$. As discussed above, the growth of excess MLG might be related to the excessive residual oxidative species in the AP-CVD system. To further improve the uniformity of BLG, the Ar flow time was increased. For the Ar-160 min
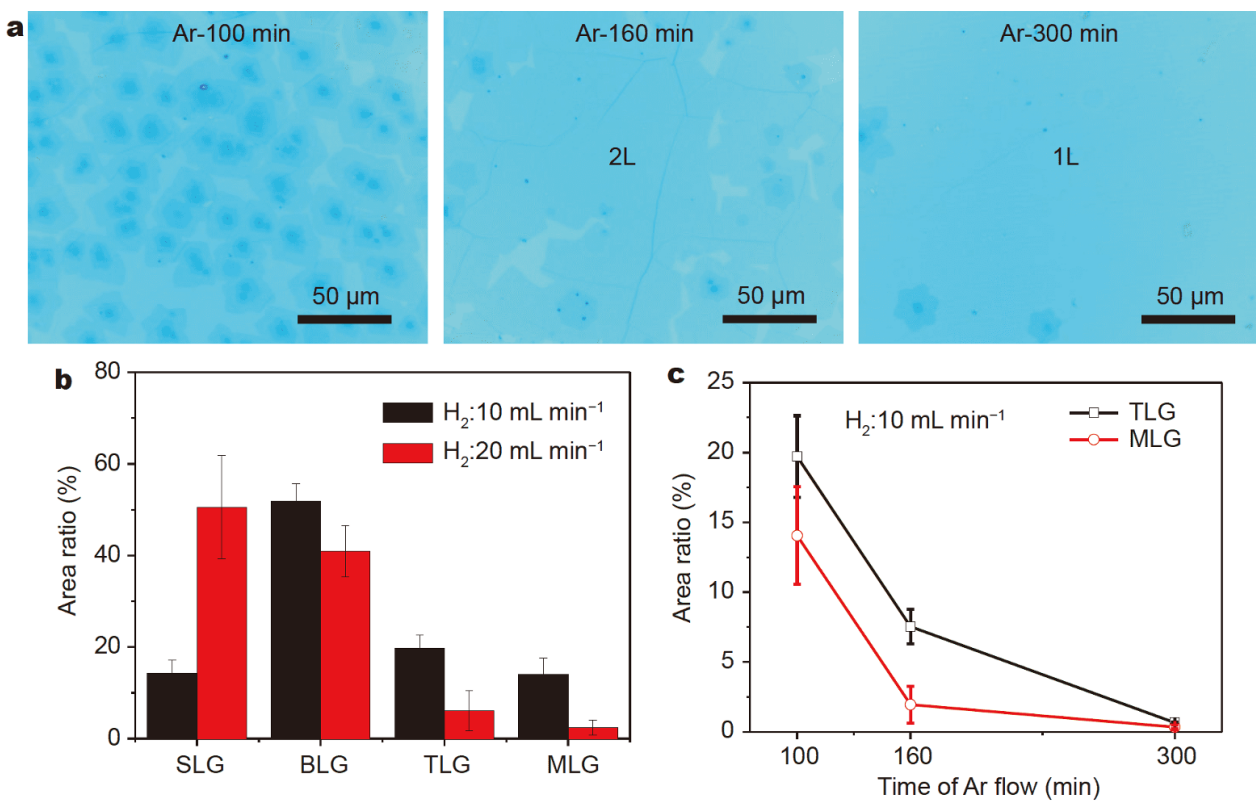

Figure 4 (a) Optical images of the top side graphene grown with 100, 160, and $300 \mathrm{~min}$ of Ar flow $\left(\mathrm{H}_{2}\right.$ flow: $\left.10 \mathrm{~mL} \mathrm{~min}^{-1}\right)$. (b) The coverage comparison of SLG, BLG, TLG, MLG with 10 and $20 \mathrm{~mL} \mathrm{~min}^{-1}$ of $\mathrm{H}_{2}$ flow (Ar flow: $100 \mathrm{~min}$ ). (c) Correlation between the coverage of TLG and MLG and the Ar flow time. 
samples, the growth of MLG was reduced, and BLG coverage in the graphene films was about $76 \%$ (Table S2). Further increasing the Ar flow time to 300 min sharply suppressed the growth of ALG, forming SLG dominated films. The area percentage of TLG and MLG also decreased with the increase of Ar flow at low hydrogen flow rates. The results further confirm the important effect of residual oxygen in the CVD system on the growth of BLG.

Therefore, the preparation of large continuous BLG films requires sufficient nucleation sites and enough growth rates. The growth kinetics is a complex interaction between carbon precursor, catalyst, oxygen and hydrogen. By adjusting the growth parameters to balance the effects of hydrogen and system residual oxygen, the productivity and uniformity of BLG can be effectively optimized. Raman mapping and TEM characterizations were conducted to demonstrate the quality of the obtained BLG. As shown in Fig. 5a, a clear straight BLG edge with optimal crystallinity is in the high-resolution TEM (HRTEM) image. Corresponding selected-area electron diffraction (SAED) pattern shown in Fig. 5b displays a typical hexagonal crystalline structure. The intensity of $\{1100\}$ diffraction spot is significantly lower than $\{2110\}$, further demonstrating the BLG feature [42]. Raman mapping was measured over a randomly selected $20 \times 20 \mathrm{~m}^{2}$, region of BLG. The corresponding optical image and typical Raman spectra are shown in Fig. S7.
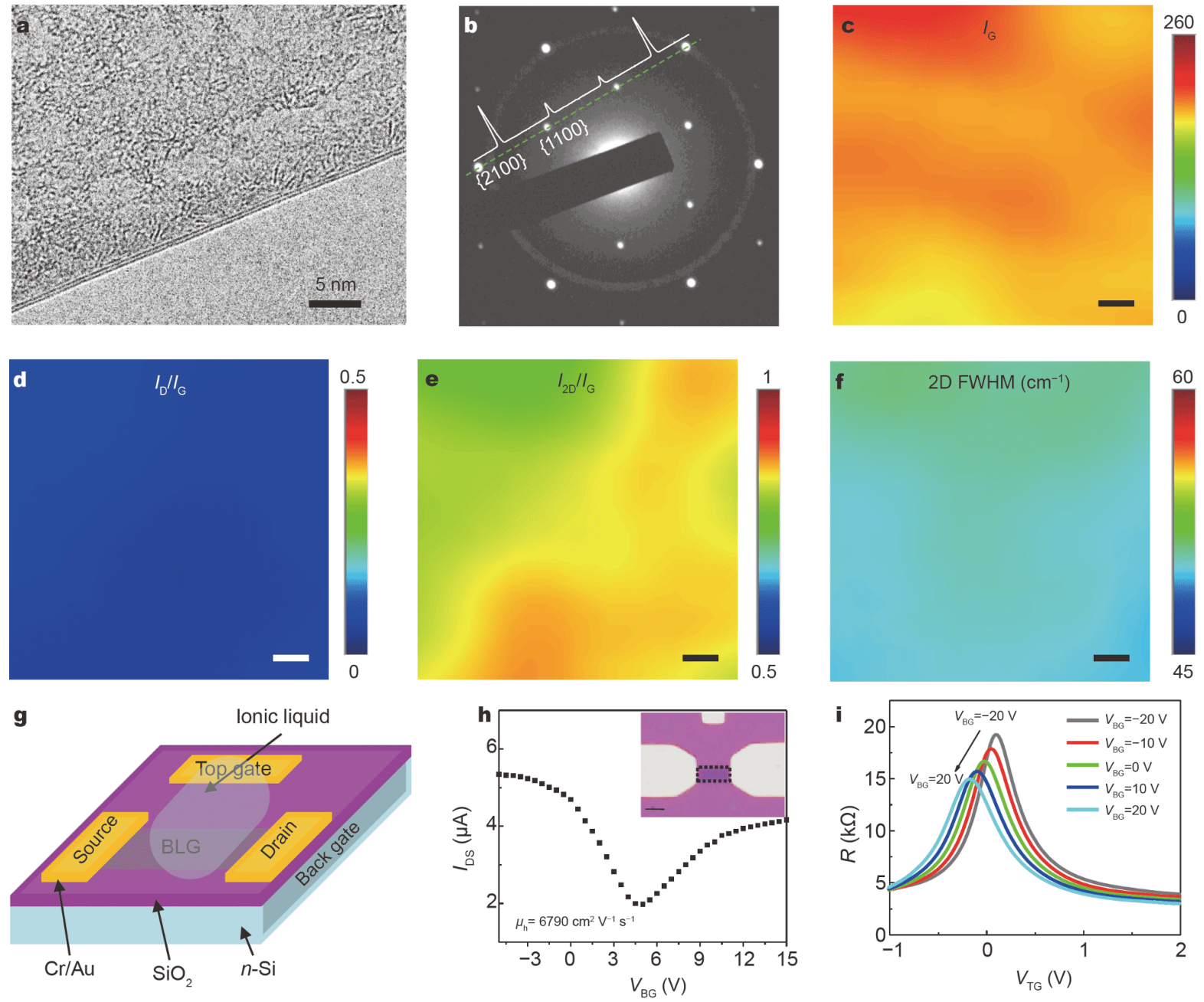

Figure 5 (a) HRTEM image and (b) SAED pattern of BLG. The inset shows the corresponding intensity profile of diffraction spots indicated by the green dash line. (c-f) Raman mappings of $G$ peak intensity, the intensity ratio of $D / G, 2 D / G$ and $2 \mathrm{D}$ peak FWHM measured over a $20 \times 20 \mu m^{2}$ region (scale bars: $2 \mu \mathrm{m}$ ). (g) Schematic illustration and (h) transport characteristic of the dual-gated BLG FET device. The inset shows the optical image of the device (scale bar: $5 \mu \mathrm{m}$ ). BLG is indicated by a black dashed square. (i) Series of the channel resistance $R$ plotted as a function of the top-gate voltage $V_{\mathrm{TG}}$ at different fixed back-gate voltages $V_{\mathrm{BG}}$ from -20 to $20 \mathrm{~V}$, with $10 \mathrm{~V}$ increment. 
The intensity of $G$ band $\left(I_{G}\right)$ is uniformly distributed around 200 in Fig. 5c, indicating an optimal continuity. The undetectable $I_{\mathrm{D}} / I_{\mathrm{G}}$ ratio shown in Fig. $5 \mathrm{~d}$ indicates a low density of defect. The $I_{2 \mathrm{D}} / I_{\mathrm{G}}$ values are mainly between 0.5 and 1 (Fig. 5e), and the FWHM of the $2 \mathrm{D}$ band in Fig. $5 \mathrm{f}$ is about $50 \mathrm{~cm}^{-1}$, corresponding to a highquality continuous $A B$ stacked BLG. To further analyze the stacking order of the BLG film, Raman measurements were conducted on randomly selected fifty BLG regions (Fig. S8). The results show that $75 \%$ of the $I_{2 \mathrm{D}} / I_{\mathrm{G}}$ ratio are $\sim 1$, and $62 \%$ of $2 \mathrm{D}$ FWHM are $\sim 50 \mathrm{~cm}^{-1}$. The scatter plot where the $y$-axis is the FWHM of the 2D peak and the $x$ axis is the $I_{2 \mathrm{D}} / I_{\mathrm{G}}$ ratio (Fig. S8d) indicates $60 \%$ of the BLG are $\mathrm{AB}$-stacked.

As shown in Fig. 5g, a BLG-based dual-gate FET was fabricated on a $300 \mathrm{~nm} \mathrm{SiO} / / \mathrm{Si}$ substrate with $\mathrm{Cr} / \mathrm{Au}$ as the source/drain electrode, $\mathrm{SiO}_{2}$ as the back-gate dielectric and ionic-liquid forming the top gate. Electrical measurements were performed on the device and Fig. $5 \mathrm{~h}$ shows plots of $I_{\mathrm{DS}}$ versus $V_{\mathrm{BG}}$ of the device. The carrier mobility reaches $6790 \mathrm{~cm}^{2} \mathrm{~V}^{-1} \mathrm{~s}^{-1}$ at room temperature, which is better than most of previously reported CVD BLG $[8,10,11,17,25,43]$ and mechanically exfoliated BLG $[1,44]\left(500\right.$ to $\left.5000 \mathrm{~cm}^{2} \mathrm{~V}^{-1} \mathrm{~s}^{-1}\right)$. A perpendicular electric field on the BLG was used to demonstrate the bandgap tunability by adjusting the applied voltages to the two gate electrodes. When sweeping the top gate voltage $\left(V_{\mathrm{TG}}\right)$ from -2 to $2 \mathrm{~V}$ with $V_{\mathrm{BG}}$ fixed between -20 to $20 \mathrm{~V}$, the channel resistance $(R)$ versus $V_{\mathrm{TG}}$ transfer curves varied with different $V_{\mathrm{BG}}$ (Fig. 5i), which was consistent with the behavior of AB stacked BLG devices $[8,10,17]$. Due to the use of ionic liquid as the top-gate dielectric, a typical gate hysteresis behavior [8] between two consecutive transfer characteristics $\left(I_{\mathrm{DS}}-V_{\mathrm{BG}}\right)$ was found in the device (Fig. S9a). This strong doping effect induced by ionic liquid can be inhibited by delaying the second transfer characteristics scanning after $1 \mathrm{~h}$ of the first scan, as shown in Fig. S9b, which demonstrates the reliability of the dual-gate devices. The characterization results reveal the high quality of the BLG prepared by AP-CVD.

\section{CONCLUSIONS}

In summary, by comprehensively understanding the growth process in AP-CVD, we developed a simple yet efficient approach to synthesize BLG on $\mathrm{Cu}$ foil which was softened and stacked onto the back-side quartz during the heating up process. The quartz significantly promoted the formation of large BLG domains on the top side of $\mathrm{Cu}$ foil. Oxidized $\mathrm{Cu}$ foil by the residual oxygen was a key accelerator for the BLG growth. Reduction with hydrogen increased the nucleation density, and thus optimized the uniformity of BLG. Finally, large area continuous graphene films with a BLG coverage of $76 \%$ were obtained by optimizing residual oxygen content and nucleation density. The BLG exhibited tunable bandgaps with high carrier mobility up to $6790 \mathrm{~cm}^{2} \mathrm{~V}^{-1} \mathrm{~s}^{-1}$ at room temperature. The investigation of BLG growth in this study will be helpful for the controllable synthesis of graphene with specific number of layers.

\section{Received 8 April 2020; accepted 13 May 2020;}

published online 14 July 2020

1 Zhang Y, Tang TT, Girit C, et al. Direct observation of a widely tunable bandgap in bilayer graphene. Nature, 2009, 459: 820-823

2 Cao Y, Fatemi V, Fang S, et al. Unconventional superconductivity in magic-angle graphene superlattices. Nature, 2018, 556: 43-50

3 Gao Y, Cao T, Cellini F, et al. Ultrahard carbon film from epitaxial two-layer graphene. Nat Nanotech, 2018, 13: 133-138

4 Li X, Cai W, An J, et al. Large-area synthesis of high-quality and uniform graphene films on copper foils. Science, 2009, 324: 13121314

5 Bae S, Kim H, Lee Y, et al. Roll-to-roll production of 30-inch graphene films for transparent electrodes. Nat Nanotech, 2010, 5: 574-578

6 Li X, Cai W, Colombo L, et al. Evolution of graphene growth on $\mathrm{Ni}$ and $\mathrm{Cu}$ by carbon isotope labeling. Nano Lett, 2009, 9: 4268-4272

7 López GA, Mittemeijer EJ. The solubility of C in solid Cu. Scripta Mater, 2004, 51: 1-5

8 Wu J, Wang J, Pan D, et al. Synchronous growth of high-quality bilayer Bernal graphene: from hexagonal single-crystal domains to wafer-scale homogeneous films. Adv Funct Mater, 2017, 27: 1605927

9 Zhou H, Yu WJ, Liu L, et al. Chemical vapour deposition growth of large single crystals of monolayer and bilayer graphene. Nat Commun, 2013, 4: 2096

10 Liu L, Zhou H, Cheng R, et al. High-yield chemical vapor deposition growth of high-quality large-area $\mathrm{AB}$-stacked bilayer graphene. ACS Nano, 2012, 6: 8241-8249

11 Qi Z, Shi H, Zhao M, et al. Chemical vapor deposition growth of bernal-stacked bilayer graphene by edge-selective etching with $\mathrm{H}_{2} \mathrm{O}$. Chem Mater, 2018, 30: 7852-7859

$12 \mathrm{Wu} \mathrm{Y}$, Chou $\mathrm{H}$, Ji H, et al. Growth mechanism and controlled synthesis of $\mathrm{AB}$-stacked bilayer graphene on $\mathrm{Cu}-\mathrm{Ni}$ alloy foils. ACS Nano, 2012, 6: 7731-7738

13 Liu W, Kraemer S, Sarkar D, et al. Controllable and rapid synthesis of high-quality and large-area Bernal stacked bilayer graphene using chemical vapor deposition. Chem Mater, 2013, 26: 907-915

14 Yang $\mathrm{C}, \mathrm{Wu} \mathrm{T}$, Wang $\mathrm{H}$, et al. Copper-vapor-assisted rapid synthesis of large $\mathrm{AB}$-stacked bilayer graphene domains on $\mathrm{Cu}-\mathrm{Ni}$ alloy. Small, 2016, 12: 2009-2013

15 Yoo MS, Lee HC, Lee S, et al. Chemical vapor deposition of Bernalstacked graphene on a $\mathrm{Cu}$ surface by breaking the carbon solubility symmetry in $\mathrm{Cu}$ foils. Adv Mater, 2017, 29: 1700753

16 Hao Y, Wang L, Liu Y, et al. Oxygen-activated growth and bandgap tunability of large single-crystal bilayer graphene. Nat Nanotech, 2016, 11: 426-431

17 Yan K, Peng H, Zhou Y, et al. Formation of bilayer Bernal gra- 
phene: layer-by-layer epitaxy via chemical vapor deposition. Nano Lett, 2011, 11: 1106-1110

18 Celebi K, Cole MT, Choi JW, et al. Evolutionary kinetics of graphene formation on copper. Nano Lett, 2013, 13: 967-974

19 Fang W, Hsu AL, Song Y, et al. Asymmetric growth of bilayer graphene on copper enclosures using low-pressure chemical vapor deposition. ACS Nano, 2014, 8: 6491-6499

$20 \mathrm{Li} \mathrm{Q}$, Chou $\mathrm{H}$, Zhong JH, et al. Growth of adlayer graphene on $\mathrm{Cu}$ studied by carbon isotope labeling. Nano Lett, 2013, 13: 486-490

21 Chan CC, Chung WL, Woon WY. Nucleation and growth kinetics of multi-layered graphene on copper substrate. Carbon, 2018, 135: 118-124

22 Shen C, Yan X, Qing F, et al. Criteria for the growth of large-area adlayer-free monolayer graphene films by chemical vapor deposition. J Materiomics, 2019, 5: 463-470

23 Abidi IH, Liu Y, Pan J, et al. Regulating top-surface multilayer/ single-crystal graphene growth by "gettering" carbon diffusion at backside of the copper foil. Adv Funct Mater, 2017, 27: 1700121

24 Han Z, Kimouche A, Kalita D, et al. Homogeneous optical and electronic properties of graphene due to the suppression of multilayer patches during CVD on copper foils. Adv Funct Mater, 2014, 24: 964-970

25 Yan Z, Liu Y, Ju L, et al. Large hexagonal bi- and trilayer graphene single crystals with varied interlayer rotations. Angew Chem Int Ed, 2014, 53: 1565-1569

26 Chen Q, Zhong Y, Huang M, et al. Direct growth of high crystallinity graphene from water-soluble polymer powders. 2D Mater, 2018, 5: 035001

27 Chen Q, Yi X, Huang M, et al. Sustained and controlled release of volatile precursors for chemical vapor deposition of graphene at atmospheric pressure. Chem Eur J, 2020, 26: 7463-7469

28 Liang T, Luan $\mathrm{C}$, Chen $\mathrm{H}$, et al. Exploring oxygen in graphene chemical vapor deposition synthesis. Nanoscale, 2017, 9: 37193735

29 Srinivasan BM, Hao Y, Hariharaputran R, et al. Oxygen-promoted chemical vapor deposition of graphene on copper: a combined modeling and experimental study. ACS Nano, 2018, 12: 9372-9380

30 Hao Y, Bharathi MS, Wang L, et al. The role of surface oxygen in the growth of large single-crystal graphene on copper. Science, 2013, 342: 720-723

$31 \mathrm{Xu} \mathrm{X}$, Zhang Z, Qiu L, et al. Ultrafast growth of single-crystal graphene assisted by a continuous oxygen supply. Nat Nanotech, 2016, 11: 930-935

32 Chen J, Cui M, Wu G, et al. Fast growth of large single-crystalline graphene assisted by sequential double oxygen passivation. Carbon, 2017, 116: 133-138

33 Ferrari AC, Basko DM. Raman spectroscopy as a versatile tool for studying the properties of graphene. Nat Nanotech, 2013, 8: 235246

34 Ferrari AC, Meyer JC, Scardaci V, et al. Raman spectrum of graphene and graphene layers. Phys Rev Lett, 2006, 97: 187401

$35 \mathrm{Wu} \mathrm{R}, \mathrm{Pan} \mathrm{J}, \mathrm{Ou} \mathrm{X}$, et al. Concurrent fast growth of sub-centimeter single-crystal graphene with controlled nucleation density in a confined channel. Nanoscale, 2017, 9: 9631-9640

36 Pang J, Bachmatiuk A, Fu L, et al. Oxidation as a means to remove surface contaminants on $\mathrm{Cu}$ foil prior to graphene growth by chemical vapor deposition. J Phys Chem C, 2015, 119: 1336313368

37 Magnuson CW, Kong X, Ji H, et al. Copper oxide as a "selfcleaning" substrate for graphene growth. J Mater Res, 2014, 29:
403-409

38 Chen X, Zhao P, Xiang R, et al. Chemical vapor deposition growth of $5 \mathrm{~mm}$ hexagonal single-crystal graphene from ethanol. Carbon, 2015, 94: 810-815

39 Wang ZJ, Dong J, Cui Y, et al. Stacking sequence and interlayer coupling in few-layer graphene revealed by in situ imaging. Nat Commun, 2016, 7: 13256

40 Wang H, Wang G, Bao P, et al. Controllable synthesis of submillimeter single-crystal monolayer graphene domains on copper foils by suppressing nucleation. J Am Chem Soc, 2012, 134: 36273630

41 Yan Z, Lin J, Peng Z, et al. Toward the synthesis of wafer-scale single-crystal graphene on copper foils. ACS Nano, 2012, 6: 91109117

42 Hernandez Y, Nicolosi V, Lotya M, et al. High-yield production of graphene by liquid-phase exfoliation of graphite. Nat Nanotech, 2008, 3: 563-568

43 Yi D, Jeon S, Hong SW. Selectively patterned regrowth of bilayer graphene for self-integrated electronics by sequential chemical vapor deposition. ACS Appl Mater Interfaces, 2018, 10: 4001440023

44 Zou K, Zhu J. Transport in gapped bilayer graphene: the role of potential fluctuations. Phys Rev B, 2010, 82: 081407

Acknowledgements This work was supported by China Postdoctoral Science Foundation (2018M642831), and Shenzhen Science and Technology Project (JCYJ20180507183904841).

Author contributions Chen Q, Wang S, and Zhu H proposed the concept and conceived the experiments. Chen Q, Song Q, Yi X, Chen Q, Wu W, Huang M, and Zhao C carried out the experiments. Chen $\mathrm{Q}$ drafted the manuscript and all authors discussed and revised it.

Conflict of interest The authors declare no conflict of interest.

Supplementary information Supporting data are available in the online version of the paper.

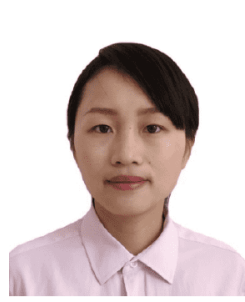

Qiao Chen received her BSc degree from Zhengzhou University in 2013. She obtained her $\mathrm{PhD}$ degree under the supervision of Prof. Hongwei Zhu in 2018 from Tsinghua University. She then worked as a postdoctoral fellow at Huazhong University of Science and Technology. Her current research interest is the design and preparation of low dimensional carbon structures by CVD.

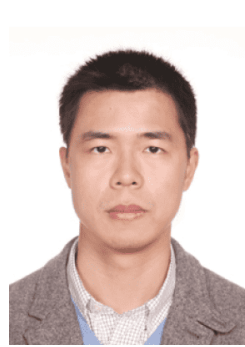

Shun Wang received his $\mathrm{PhD}$ degree from the University of Minnesota in 2010. He then worked as a postdoctoral researcher at the University of Minnesota and as an associate professor at Shanghai Jiao Tong University. Currently he is a professor at Huazhong University of Science and Technology. His research interests are the electrical and optoelectronic properties of low dimensional materials. 


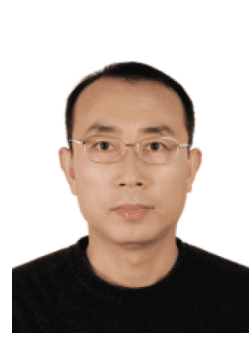

Hongwei Zhu is a professor of the School of Materials Science and Engineering, Tsinghua University. He received his BSc degree in mechanical engineering (1998) and $\mathrm{PhD}$ degree in materials processing engineering (2003) from Tsinghua University. After postdoc experience in Japan and USA, he began his independent career as a faculty member at Tsinghua University (2008-present). His current research interests involve the structural design and engineering of nanomaterials for energy and environmental applications.
常压化学气相沉积法在软化铜箔上生长高质量双 层石墨烯

陈巧 ${ }^{1,2}$, 宋启扬 ${ }^{1}$, 易新 ${ }^{1}$, 陈乔 ${ }^{1}$, 吴文嘉 ${ }^{1}$, 黄美榕 ${ }^{2}$, 赵传文 ${ }^{1}$, 王顺 ${ }^{13^{*}}$, 朱宏伟 ${ }^{2 *}$

摘要 双层石墨烯因其独特的物理性质在新型电子器件等领域具 有广阔的应用前景. 大面积高质量双层石墨烯的批量化制备是实 现其后续应用的关键. 目前, 基于铜表面自限制催化的化学气相沉 积法可有效实现单层石墨烯的生长, 但由于第二层石墨烯结构导 致更复杂的生长过程, 双层石墨烯的可控制备极具挑战性. 本文系 统研究了石墨烯的常压化学气相沉积制备过程, 提出了一种在软 化铜䇴基底上生长高质量双层石墨烯的方法. 铜管在随炉升温过 程中软化并贴合至背面的石英舟/管表面, 形成具有差异反应条件 的铜箔双面，从而促使双层石墨烯在其正面生长. 反应系统中的残 余氧气有效加快了双层石墨烯的生长. 同时, 适量的残余氧气可抑 制三层及少数层石墨烯的形成, 提高双层石墨烯产物的均匀性. 基 于优化的生长条件, 在 $4 \times 4 \mathrm{~cm}^{2}$ 铜管上实现了双层覆盖率达 $76 \%$ 的 高质量石墨烯薄膜的生长. 基于 $\mathrm{AB}$ 堆垛双层石墨烯的双栅场效应 晶体管, 室温载流子迁移率达 $6790 \mathrm{~cm}^{2} \mathrm{~V}^{-1} \mathrm{~s}^{-1}$. 本研究有助于推动 石墨烯等二维材料的层数可控合成技术的发展. 